\title{
Three positive solutions for second-order periodic boundary value problems with sign-changing weight
}

Zhiqian $\mathrm{He}^{1}$, Ruyun $\mathrm{Ma}^{1 *}$ and Man $\mathrm{Xu}^{1}$

\section{"Correspondence:}

mary@nwnu.edu.cn

${ }^{1}$ Department of Mathematics,

Northwest Normal University,

Lanzhou, P.R. China

\begin{abstract}
In this paper, we study the global structure of positive solutions of periodic boundary value problems

$$
\left\{\begin{array}{l}
-u^{\prime \prime}(t)+q(t) u(t)=\lambda h(t) f(u(t)), \quad t \in(0,2 \pi), \\
u(0)=u(2 \pi), \quad u^{\prime}(0)=u^{\prime}(2 \pi)
\end{array}\right.
$$

where $q \in C([0,2 \pi],[0,+\infty))$ with $q \neq \equiv, f \in C(\mathbb{R}, \mathbb{R})$, the weight $h \in C[0,2 \pi]$ is a sign-changing function, $\lambda$ is a parameter. We prove the existence of three positive solutions when $h(t)$ has $n$ positive humps separated by $n+1$ negative ones. The proof is based on the bifurcation method.
\end{abstract}

MSC: 34B10; 34B18

Keywords: Three positive solutions; Periodic boundary value problem; Bifurcation

\section{Introduction}

In this paper, we study the global structure of positive solutions of periodic boundary value problems (PBVPs)

$$
\left\{\begin{array}{l}
-u^{\prime \prime}(t)+q(t) u(t)=\lambda h(t) f(u(t)), \quad t \in(0,2 \pi), \\
u(0)=u(2 \pi), \quad u^{\prime}(0)=u^{\prime}(2 \pi),
\end{array}\right.
$$

where $q \in C([0,2 \pi],[0, \infty))$ with $q \neq \equiv 0, f \in C(\mathbb{R}, \mathbb{R}), \lambda$ is a parameter, and $h \in C[0,2 \pi]$ satisfies the following condition:

(F1) There exist $x_{1}, x_{2}, \ldots, x_{2 n} \in[0,2 \pi]$ with $x_{1}<x_{2}<\cdots<x_{2 n}$ such that $h(t)>0$ on $\left(x_{2 i-1}, x_{2 i}\right)$, and $h(t)<0$ on $[0,2 \pi] \backslash\left[x_{2 i-1}, x_{2 i}\right], i=1, \ldots, n$.

In the recent years, PBVPs have been studied by many authors; see [1-13] and the references therein. In particular, we refer to the papers of J. R. Graef et al. [1] and Hao et al. [2]. In these works, the authors established some results of existence and multiplicity of positive solutions for problem (1.1) with sign-definite weight, and, in general, the main tool in the proofs is the fixed point index theory in cones. Clearly, this theory is no longer available if the weight function changes its sign. However, to our knowledge, problem (1.1)

(c) The Author(s) 2018. This article is distributed under the terms of the Creative Commons Attribution 4.0 International License (http://creativecommons.org/licenses/by/4.0/), which permits unrestricted use, distribution, and reproduction in any medium, provided you give appropriate credit to the original author(s) and the source, provide a link to the Creative Commons license, and indicate if changes were made. 
with sign-changing weight, in spite of its simple looking structure, is considered as a hard problem in the literature due to the lack of any a priori estimate over the set of possible periodic solutions. In order to overcome this difficulty, Ma et al. [3] used a different method, so-called bifurcation; their results extend and improve the corresponding results of $[1,2]$.

Recently, there has been a lot of works concerning superlinear/sublinear problems with sign-changing weight, we refer the reader to $[4,5]$ and the references therein. In [5], by using Leray-Schauder degree theory, Hakl and Zamora established efficient conditions to guarantee the existence of a $T$-periodic solution to the second order differential equation

$$
u^{\prime \prime}(t)=h(t) g(u(t))
$$

where $h \in L(\mathbb{R} / T \mathbb{Z})$ and $h(\cdot)$ changes its sign, and $\bar{h}:=\int_{0}^{T} h(s) d s<0, g \in C^{1}\left(\mathbb{R}^{+} ; \mathbb{R}^{+}\right)$is a nonincreasing function with a strong singularity at zero, i.e.,

$$
\lim _{x \rightarrow 0^{+}} \int_{x}^{1} g(s) d s=+\infty
$$

It is worth noting that they cannot obtain three positive solutions, and even cannot guarantee the positivity of all solutions.

Compared with the above works dealing with the study of semilinear problems, Dai et al. [6] studied a unilateral global bifurcation result for a class of quasilinear PBVPs

$$
\left\{\begin{array}{l}
-\left(\varphi_{p}\left(u^{\prime}\right)\right)^{\prime}+q(t) \varphi_{p}(u)=\lambda m(t) f(u), \quad t \in(0, T), \\
u(0)=u(T), \quad u^{\prime}(0)=u^{\prime}(T),
\end{array}\right.
$$

where $1<p<\infty, \varphi_{p}(s)=|s|^{p-2} s, q \in C([0, T],[0, \infty))$ with $q \not \equiv 0, f \in C(\mathbb{R}, \mathbb{R}), \lambda$ is a parameter. By virtue of bifurcation techniques, they established some results of existence of one-sign solutions for problem (1.3) according to the asymptotic behavior of $f$ at 0 and $\infty$. However, the sublinear and superlinear conditions imposed on the nonlinearities only deduce a relatively simple "shape of the component", and they provided no information on at least two direction turns of the connected component. Recently, there has been a result about one-dimensional $p$-Laplacian problem by Sim and Tanaka [14]:

$$
\left\{\begin{array}{l}
-\left(\left|u^{\prime}\right|^{p-2} u^{\prime}\right)^{\prime}=\lambda h(t) f(u), \quad t \in(0,1), \\
u(0)=u(1)=0
\end{array}\right.
$$

where $p>1, \lambda>0$ is a parameter and the weight function $h$ satisfies:

(A1) There exist $x_{1}, x_{2} \in[0,1]$ such that $x_{1}<x_{2}, h(t)>0$ on $\left(x_{1}, x_{2}\right)$ and $h(t) \leq 0$ on $[0,1] \backslash\left[x_{1}, x_{2}\right]$.

Based upon bifurcation method, they showed that (1.4) has three positive solutions suggesting suitable conditions on the weight function and nonlinearity.

Motivated by these studies, we are interested in investigating the shape of unbounded continua of solutions. Moreover, we show the existence and multiplicity of positive solutions with respect to parameter $\lambda$ by figuring the shape of continua of solutions, and especially, we obtain the existence of three positive solutions for $\lambda$ being in a certain interval. 
Throughout the paper, we always suppose that $f$ satisfies the following signum condition:

(H0) $f \in C^{2}(\mathbb{R}, \mathbb{R})$ with $f(s) s>0$ for $s \neq 0$.

Clearly, (H0) implies $f(0)=0$, hence, $u=0$ is always the solution of problem (1.1). In order to study the global bifurcation phenomena of problem (1.1), we must consider the following eigenvalue problem:

$$
\left\{\begin{array}{l}
-u^{\prime \prime}(t)+q(t) u(t)=\lambda h(t) u(t), \quad t \in(0,2 \pi) \\
u(0)=u(2 \pi), \quad u^{\prime}(0)=u^{\prime}(2 \pi)
\end{array}\right.
$$

In 1997, Constantin [7] proved that problem (1.5) possesses two infinite sequences of eigenvalues

$$
\cdots<\lambda_{2}^{-} \leq \lambda_{1}^{-}<\lambda_{0}^{-}<0<\lambda_{0}^{+}<\lambda_{1}^{+} \leq \lambda_{2}^{+} \cdots
$$

such that $\lambda_{0}^{+}$and $\lambda_{0}^{-}$are simple eigenvalues with positive eigenfunctions.

Remark 1.1 The eigenvalues $\lambda_{0}^{+}, \lambda_{0}^{-}$are the minimum and maximum of the "Rayleigh quotient" respectively, that is,

$$
\lambda_{0}^{+}=\inf \left\{\int_{0}^{2 \pi}\left(\left(v^{\prime}\right)^{2}+q v^{2}\right) d t \mid v(0)=v(2 \pi), v^{\prime}(0)=v^{\prime}(2 \pi), \int_{0}^{2 \pi} h v^{2} d t=1\right\}
$$

and

$$
\lambda_{0}^{-}=\sup \left\{-\int_{0}^{2 \pi}\left(\left(v^{\prime}\right)^{2}+q v^{2}\right) d t \mid v(0)=v(2 \pi), v^{\prime}(0)=v^{\prime}(2 \pi), \int_{0}^{2 \pi}-h v^{2} d t=1\right\} .
$$

(H1) There exist constants $f_{0}, \delta, \hat{\delta} \in(0, \infty)$ such that

$$
\begin{aligned}
& f(s)=f_{0} s-g(s), \quad s \in[0, \delta) . \\
& g(s)>0 \quad \text { for all } s>0, \quad \lim _{s \rightarrow 0^{+}} \frac{g(s)}{s}=0, \\
& \lim _{c \rightarrow 0^{+}} \int_{0}^{2 \pi} h(t) \phi(t) \frac{g(c \phi(t))}{c \ln (1+c)} d t=\hat{\delta}>0,
\end{aligned}
$$

where $\phi$ is the positive eigenfunction corresponding to $\lambda_{0}^{+}$;

(H2) $f_{\infty}:=\lim _{s \rightarrow \infty} \frac{f(s)}{s}=0$;

(H3) There exists $s_{0}>0$ such that

$$
\min _{s \in\left[s_{0}, 2 s_{0}\right]} \frac{f(s)}{s} \geq \frac{f_{0}}{\lambda_{0}^{+} h_{0}}\left[\left(\frac{2 \pi}{l}\right)^{2}+\hat{q}\right],
$$

where $l=\min _{1 \leq i \leq n}\left\{x_{2 i}-x_{2 i-1}\right\}, \hat{q}=\max _{s \in[0,2 \pi]} q(s)$,

$h_{0}=\min _{1 \leq i \leq n}\left\{h(t) \mid t \in\left[\frac{t_{0}+x_{2 i-1}}{2}, \frac{t_{0}+x_{2 i}}{2}\right]\right\}$ for some $t_{0} \in\left(x_{2 i-1}, x_{2 i}\right)$; 
(H4) There exists $\beta>0$ such that

$$
\beta h^{+}(t) \inf \left\{\frac{f(s)}{s}: s \in\left(0,2 s_{0}\right]\right\} \geq q(t), \quad t \in \bigcup_{i=1}^{n}\left(x_{2 i-1}, x_{2 i}\right),
$$

where $h^{+}(t):=\max \{h(t), 0\}$

(H5) $q \in C[0,2 \pi]$ with $q \geq 0$ and $q(t) \not \equiv 0$ in $[0,2 \pi]$ satisfies

$$
8 \pi^{2} s_{0}\|q\|_{\infty}+2 \pi \beta\left\|h^{-}\right\|_{L^{1}(0,2 \pi)} \max \left\{f(s): s \in\left[0,2 s_{0}\right]\right\} \leq s_{0},
$$

where $h^{-}(t):=\max \{-h(t), 0\}$.

It is easy to find that if $(\mathrm{H} 1)$ holds, then

$$
\lim _{s \rightarrow 0^{+}} \frac{f(s)}{s}=f_{0} .
$$

Moreover, if (1.6) and (H2) hold, then there exists $N>0$ such that

$$
f(s) \leq N s, \quad s \geq 0 .
$$

Theorem 1.1 Assume that (F1), (H0)-(H3) hold. Then there exist $\lambda_{*} \in\left(0, \frac{\lambda_{0}^{+}}{f_{0}}\right)$ and $\lambda^{*}>\frac{\lambda_{0}^{+}}{f_{0}}$ such that

(i) (1.1) has at least one positive solution if $\lambda=\lambda_{*}$;

(ii) (1.1) has at least two positive solutions if $\lambda_{*}<\lambda \leq \frac{\lambda_{0}^{+}}{f_{0}}$;

(iii) (1.1) has at least three positive solutions if $\frac{\lambda_{0}^{+}}{f_{0}}<\lambda<\lambda^{*}$;

(iv) (1.1) has at least two positive solutions if $\lambda=\lambda^{*}$;

(v) (1.1) has at least one positive solution if $\lambda>\lambda^{*}$;

(vi) (1.1) has at least one positive solution if $\lambda<\frac{\lambda_{0}^{-}}{f_{0}}$.

Remark 1.2 Condition (F1) implies that the weight function $h$ has $n$ positive humps separated by $n+1$ negative ones. Clearly, when $n=1$, our condition (F1) will reduce to (A1). Moreover, we do not require that the mean value of $h$ is definite.

An outline of the work is as follows. In Sect. 2, we show global bifurcation phenomena from the trivial branch with the rightward direction near the initial point. Section 3 is devoted to showing the change of direction of bifurcation and to completing the proof of Theorem 1.1.

\section{Preliminaries and rightward bifurcation}

In this section, we state some preliminary results and show global bifurcation phenomena from the trivial branch with the rightward direction.

Let $G(t, s)$ be the Green's function of the homogeneous PBVPs

$$
\left\{\begin{array}{l}
-u^{\prime \prime}(t)+q(t) u(t)=0, \quad t \in(0,2 \pi) \\
u(0)=u(2 \pi), \quad u^{\prime}(0)=u^{\prime}(2 \pi)
\end{array}\right.
$$


From Theorem 2.5 of [10], we know that $G(t, s)>0, \forall t, s \in[0,2 \pi]$.

Let $Y=C[0,2 \pi]$ with the norm

$$
\|u\|_{\infty}=\max _{t \in[0,2 \pi]}|u(t)|
$$

$E=\left\{u \in C^{1}[0,2 \pi]: u(0)=u(2 \pi), u^{\prime}(0)=u^{\prime}(2 \pi)\right\}$ with the norm

$$
\|u\|=\max _{t \in[0,2 \pi]}|u(t)|+\max _{t \in[0,2 \pi]}\left|u^{\prime}(t)\right|,
$$

and $P=\{u \in E: u(t) \geq 0\}$ be the positive cone in $E$.

Define $L: D(L) \rightarrow Y$ by setting

$$
L u:=-u^{\prime \prime}+q(t) u, \quad u \in D(L),
$$

where

$$
D(L)=\left\{u \in C^{2}[0,2 \pi]: u(0)=u(2 \pi), u^{\prime}(0)=u^{\prime}(2 \pi)\right\} .
$$

Then $L^{-1}: Y \rightarrow E$ is compact.

Let $\xi, \zeta \in C(\mathbb{R}, \mathbb{R})$ be such that $f(u)=f_{0} u+\xi(u), f(u)=f_{\infty} u+\zeta(u)$. Clearly,

$$
\lim _{u \rightarrow 0} \frac{\xi(u)}{u}=0, \quad \lim _{u \rightarrow \infty} \frac{\zeta(u)}{u}=0 .
$$

Let $\tilde{\zeta}(u)=\max _{0 \leq s \leq u}|\zeta(s)|$, then $\tilde{\zeta}$ is nondecreasing and

$$
\lim _{u \rightarrow \infty} \frac{\tilde{\zeta}(u)}{u}=0 .
$$

Let us consider

$$
L u-\lambda h(t) f_{0} u=\lambda h(t) \xi(u)
$$

as a bifurcation problem from the trivial solution $u \equiv 0$.

Equation (2.3) can be converted to the equivalent equation

$$
\begin{aligned}
u(t) & =\int_{0}^{2 \pi} G(t, s)\left[\lambda h(s) f_{0} u(s)+\lambda h(s) \xi(u(s))\right] d s \\
& :=\lambda L^{-1}\left[h(\cdot) f_{0} u(\cdot)\right](t)+\lambda L^{-1}[h(\cdot) \xi(u(\cdot))](t) .
\end{aligned}
$$

Further we note that $\left\|L^{-1}[h(\cdot) \xi(u(\cdot))]\right\|=o(\|u\|)$ for $u$ near 0 in $E$, since

$$
\begin{aligned}
\left\|L^{-1}[h(\cdot) \xi(u(\cdot))]\right\|= & \max _{t \in[0,2 \pi]}\left|\int_{0}^{2 \pi} G(t, s) h(s) \xi(u(s)) d s\right| \\
& +\max _{t \in[0,2 \pi]}\left|\int_{0}^{2 \pi} G_{t}(t, s) h(s) \xi(u(s)) d s\right| \\
\leq & C \cdot \max _{t \in[0,2 \pi]}|h(s)| \cdot\|\xi(u(\cdot))\|_{\infty} .
\end{aligned}
$$


By an argument similar to proving [6, Theorem 4.3] with obvious changes, we may obtain the following result.

Lemma 2.1 Assume that (H0), (H1), and (F1) hold. The pair $\left(\frac{\lambda_{0}^{v}}{f_{0}}, 0\right)$ is a bifurcation point of problem (1.1). Moreover, there is an unbounded component $\mathcal{C}^{v}$ of the set of solutions of problem (1.1) and

$$
\mathcal{C}^{v} \subset\left(\operatorname{int} P \cup\left\{\left(\frac{\lambda_{0}^{v}}{f_{0}}, 0\right)\right\}\right)
$$

where $v \in\{+,-\}$.

Lemma 2.2 Assume that (F1) and (H1) hold. Let $\left\{\left(\lambda_{n}, u_{n}\right)\right\}$ be a sequence of positive solutions to (1.1) which satisfies $\lambda_{n} \rightarrow \frac{\lambda_{0}^{+}}{f_{0}}$ and $\left\|u_{n}\right\| \rightarrow 0$. Let $\phi$ be a positive eigenfunction corresponding to $\lambda_{0}^{+}$, which satisfies $\|\phi\|=1$. Then there exists a subsequence of $\left\{u_{n}\right\}$, again denoted by $\left\{u_{n}\right\}$, such that $\frac{u_{n}}{\left\|u_{n}\right\|}$ converges uniformly to $\phi$ on $[0,2 \pi]$.

Proof Set $v_{n}:=\frac{u_{n}}{\left\|u_{n}\right\|}$, since $v_{n}$ is bounded in $C^{2}[0,2 \pi]$, after taking a subsequence if necessary, we have that $\left\{v_{n}\right\}$ uniformly converges to a limit $v \in E$ with $\|v\|=1$, and we again denote by $\left\{v_{n}\right\}$ the subsequence.

For every $\left(\lambda_{n}, u_{n}\right)$, we have

$$
u_{n}(t)=\lambda_{n} \int_{0}^{2 \pi} G(t, s) h(s)\left[f_{0} u_{n}(s)+\xi\left(u_{n}(s)\right)\right] d s
$$

Dividing both sides of (2.4) by $\left\|u_{n}\right\|$, we get

$$
v_{n}(t)=\lambda_{n} \int_{0}^{2 \pi} G(t, s) h(s)\left[f_{0} v_{n}(s)+\frac{\xi\left(u_{n}(s)\right)}{\left\|u_{n}\right\|}\right] d s
$$

Since $u_{n}(s) \rightarrow 0$ for all $s \in[0,2 \pi]$, we conclude that $\frac{\xi\left(u_{n}(s)\right)}{\left\|u_{n}\right\|} \rightarrow 0$ for each fixed $s \in[0,2 \pi]$.

Lebesgue's dominated convergence theorem shows that

$$
v(t)=\lambda_{0}^{+} \int_{0}^{2 \pi} G(t, s) h(s) v(s) d s
$$

for each fixed $t \in[0,2 \pi]$, which means that $v$ is a nontrivial solution of (1.5) with $\lambda=\lambda_{0}^{+}$, and hence $v \equiv \phi$.

Next, we give an important lemma which will be used later.

Lemma 2.3 Assume that (F1) holds. Let $\alpha \geq 0$, and let $\phi$ be a positive eigenfunction corresponding to $\lambda_{0}^{+}$. Then

$$
\int_{0}^{2 \pi} h(t)[\phi(t)]^{2+\alpha} d t>0
$$


Proof Multiplying the equation of (1.5) by $\phi^{\alpha+1}$ and integrating it over $[0,2 \pi]$, we obtain

$$
\begin{aligned}
\lambda_{0}^{+} \int_{0}^{2 \pi} h(t)[\phi(t)]^{\alpha+2} d t & =-\int_{0}^{2 \pi} \phi^{\prime \prime}(t)[\phi(t)]^{\alpha+1} d t+\int_{0}^{2 \pi} q(t)[\phi(t)]^{\alpha+2} d t \\
& =(\alpha+1) \int_{0}^{2 \pi}\left[\phi^{\prime}(t)\right]^{2}[\phi(t)]^{\alpha} d t+\int_{0}^{2 \pi} q(t)[\phi(t)]^{\alpha+2} d t>0 .
\end{aligned}
$$

Lemma 2.4 Assume that (F1), (H1), and (H2) hold. Let $\mathcal{C}^{+}$be as in Lemma 2.1. Then $(\lambda, u) \in \mathcal{C}^{+}$and $\left|\lambda-\frac{\lambda_{0}^{+}}{f_{0}}\right|+\|u\| \leq \frac{\delta}{2}$ imply $\lambda>\frac{\lambda_{0}^{+}}{f_{0}}$.

Proof We divide the proof into two steps.

Step 1: We show that for $(\lambda, u) \in \mathcal{C}^{+}$satisfying $\left|\lambda-\frac{\lambda_{0}^{+}}{f_{0}}\right|+\|u\| \leq \frac{\delta}{2}$, we get $\lambda \geq \frac{\lambda_{0}^{+}}{f_{0}}$.

Assume to the contrary that there exists a sequence $\left\{\left(\lambda_{n}, u_{n}\right)\right\} \subset \mathcal{C}^{+}$such that $\lambda_{n} \rightarrow \frac{\lambda_{0}^{+}}{f_{0}}$, $\left\|u_{n}\right\| \rightarrow 0$, and $\lambda_{n}<\frac{\lambda_{0}^{+}}{f_{0}}$. By Lemma 2.2, there exists a subsequence of $\left\{u_{n}\right\}$, again denoted by $\left\{u_{n}\right\}$, such that $\frac{u_{n}}{\left\|u_{n}\right\|}$ converges uniformly to $\phi$ on $[0,2 \pi]$, where $\phi$ is the positive eigenfunction corresponding to $\lambda_{0}^{+}$, which satisfies $\|\phi\|=1$. Multiplying equation (1.1) with $(\lambda, u)=\left(\lambda_{n}, u_{n}\right)$ by $u_{n}$ and integrating it over $[0,2 \pi]$, we obtain

$$
\lambda_{n} \int_{0}^{2 \pi} h(s) f\left(u_{n}(s)\right) u_{n}(s) d s=\int_{0}^{2 \pi}\left[-u_{n}^{\prime \prime}(s)+q(s) u_{n}(s)\right] u_{n}(s) d s .
$$

By simple computation and using the definition of $\lambda_{0}^{+}$in Remark 1.1, we get

$$
\begin{aligned}
\lambda_{n} \int_{0}^{2 \pi} h(s) f\left(u_{n}(s)\right) u_{n}(s) d s & =\int_{0}^{2 \pi}\left[u_{n}^{\prime}(s)\right]^{2} d s+\int_{0}^{2 \pi} q(s) u_{n}^{2}(s) d s \\
& \geq \lambda_{0}^{+} \int_{0}^{2 \pi} h(s) u_{n}^{2}(s) d s
\end{aligned}
$$

that is,

$$
\int_{0}^{2 \pi} h(s) \frac{f\left(u_{n}(s)\right)-f_{0} u_{n}(s)}{u_{n}(s)} \frac{\left[u_{n}(s)\right]^{2}}{\left\|u_{n}\right\|^{2}} d s \geq \frac{\lambda_{0}^{+}-f_{0} \lambda_{n}}{\lambda_{n}} \int_{0}^{2 \pi} h(s) \frac{\left[u_{n}(s)\right]^{2}}{\left\|u_{n}\right\|^{2}} d s .
$$

Lebesgue's dominated convergence theorem, condition (H1), and Lemma 2.3 imply that

$$
\int_{0}^{2 \pi} h(s) \frac{f\left(u_{n}(s)\right)-f_{0} u_{n}(s)}{u_{n}(s)} \frac{\left[u_{n}(s)\right]^{2}}{\left\|u_{n}\right\|^{2}} d s \rightarrow 0 \cdot \int_{0}^{2 \pi} h(s) \phi^{2}(s) d s=0,
$$

and

$$
\int_{0}^{2 \pi} h(s) \frac{\left[u_{n}(s)\right]^{2}}{\left\|u_{n}\right\|^{2}} d s \rightarrow \int_{0}^{2 \pi} h(s) \phi^{2}(s) d s>0 .
$$

This contradicts $\lambda_{n}<\frac{\lambda_{0}^{+}}{f_{0}}$.

Step 2: We show that for all $(\lambda, u) \in \mathcal{C}^{+}$and $\left|\lambda-\frac{\lambda_{0}^{+}}{f_{0}}\right|+\|u\| \leq \frac{\delta}{2}$, we have $\lambda>\frac{\lambda_{0}^{+}}{f_{0}}$.

Let $X=\left\{u \in C^{2}[0,2 \pi]: u(0)=u(2 \pi), u^{\prime}(0)=u^{\prime}(2 \pi)\right\}, Y=C[0,2 \pi]$.

Define $F: \mathbb{R} \times X \rightarrow Y$ by

$$
F(\lambda, u):=-u^{\prime \prime}+q(t) u-\lambda h(t) f(u)
$$


Obviously,

$$
F(\lambda, 0)=0, \quad \lambda \in \mathbb{R},
$$

and $F_{u}, F_{\lambda}, F_{\lambda u}$ are continuous,

$$
F_{u}(\lambda, 0) w=-w^{\prime \prime}+q(t) w-\lambda f_{0} h(t) w
$$

and

$$
N\left(F_{u}\left(\frac{\lambda_{0}^{+}}{f_{0}}, 0\right)\right)=\operatorname{span}\{\phi\}, \quad R\left(F_{u}\left(\frac{\lambda_{0}^{+}}{f_{0}}, 0\right)\right)=\left\{v \in Y: \int_{0}^{2 \pi} v \phi d x=0\right\} .
$$

Here, $\lambda_{0}^{+}$is the first eigenvalue of problem (1.5), $\phi$ is the positive eigenfunction corresponding to $\lambda_{0}^{+}$. Thus

$$
\operatorname{dim} N\left(F_{u}\left(\frac{\lambda_{0}^{+}}{f_{0}}, 0\right)\right)=\operatorname{codim} R\left(F_{u}\left(\frac{\lambda_{0}^{+}}{f_{0}}, 0\right)\right)=1 .
$$

Since $F_{\lambda u}\left(\frac{\lambda_{0}^{+}}{f_{0}}, 0\right) \phi=-f_{0} h(t) \phi$, we have from Lemma 2.3 that

$$
f_{0} \int_{0}^{2 \pi} h(t) \phi^{2} d t>0
$$

and accordingly $F_{\lambda u}\left(\frac{\lambda_{0}^{+}}{f_{0}}, 0\right) \phi \notin R\left(F_{u}\left(\frac{\lambda_{0}^{+}}{f_{0}}, 0\right)\right)$.

Now, we are in a position to use the well-known Crandall-Rabinowitz bifurcation theorem (see [15, Theorem 1.7]). Thus, there exists a nontrivial continuously differentiable curve passing through $\left(\frac{\lambda_{0}^{+}}{f_{0}}, 0\right)$ with the form

$$
\left\{\left(\frac{\lambda_{0}^{+}}{f_{0}}(s), u(s)\right) \mid s \in(-a, a)\right\}
$$

such that

$$
\left(\frac{\lambda_{0}^{+}}{f_{0}}(s), u(s)\right)=\left(\frac{\lambda_{0}^{+}}{f_{0}}+\mu(s), s(\phi+\gamma(s))\right),
$$

where $\mu:(-a, a) \rightarrow \mathbb{R}, \gamma:(-a, a) \rightarrow Z$ are continuous functions satisfying $\mu(0)=0, \gamma(0)=$ $0, \gamma^{\prime}(0)=0$. Here, $Z$ is any complement of $N(F,(0,0))$ in $X$.

Assume to the contrary that there exists a sequence $\left\{\left(\lambda_{n}, u_{n}\right)\right\} \subset \mathcal{C}^{+}$such that $\lambda_{n}=$ $\frac{\lambda_{0}^{+}}{f_{0}}, u_{n}(t)=c_{n}\left(\phi(t)+\gamma\left(c_{n}\right)\right), t \in[0,2 \pi]$ with $\left\|u_{n}\right\| \rightarrow 0$, where $c_{n}>0, \lim _{n \rightarrow \infty} c_{n}=0$, $\lim _{n \rightarrow \infty} \frac{\gamma\left(c_{n}\right)}{c_{n}}=0$. Multiplying equation (1.1) with $(\lambda, u)=\left(\lambda_{n}, u_{n}\right)$ by $\phi(t)$ and integrating it over $[0,2 \pi]$, we obtain

$$
\int_{0}^{2 \pi}\left(\phi^{\prime \prime}(t)-q(t) \phi(t)+\lambda_{0}^{+} h(t) \phi(t)\right) u_{n}(t) d t=\lambda_{n} \int_{0}^{2 \pi} h(t) g\left(c_{n}\left(\phi(t)+\gamma\left(c_{n}\right)\right)\right) \phi(t) d t .
$$

By this fact together with (1.5), we have

$$
\int_{0}^{2 \pi}\left(\phi^{\prime \prime}(t)-q(t) \phi(t)+\lambda_{0}^{+} h(t) \phi(t)\right) u_{n}(t) d t=0,
$$


and accordingly,

$$
\int_{0}^{2 \pi} h(t) g\left(c_{n}\left(\phi(t)+\gamma\left(c_{n}\right)\right)\right) \phi(t) d t=0 .
$$

Define

$$
\begin{aligned}
& I_{n}=\int_{0}^{2 \pi} h(t) \phi(t) g\left(c_{n} \phi(t)\right) d t, \\
& J_{n}=\int_{0}^{2 \pi} h(t) \phi(t) g\left(c_{n}\left(\phi(t)+\gamma\left(c_{n}\right)\right)\right) d t .
\end{aligned}
$$

We may assume that

$$
\left|c_{n}\right| \leq 1, \quad\left|\gamma\left(c_{n}\right)\right| \leq M_{0}
$$

Let $I_{0}:=\left[0,\|\phi\|_{\infty}+M_{0}\right]$. We only need to work for the function $\left.g\right|_{I_{0}}$.

By simple computation, we get

$$
\begin{aligned}
\left|\frac{J_{n}-I_{n}}{c_{n}}\right| & \leq \frac{1}{c_{n} \ln \left(1+c_{n}\right)} \int_{0}^{2 \pi}\left|h(t) \phi(t)\left(g\left(c_{n}\left(\phi(t)+\gamma\left(c_{n}\right)\right)\right)-g\left(c_{n} \phi(t)\right)\right)\right| d t \\
& \leq \frac{\left|\gamma\left(c_{n}\right)\right|}{\ln \left(1+c_{n}\right)} 2 \pi\|h\|_{\infty}\|\phi\|_{\infty}\left\|g^{\prime}\right\|_{L^{\infty}\left(I_{0}\right)} .
\end{aligned}
$$

Here, $g^{\prime}(s)$ to the derivative of $g(s)$. Moreover, $\gamma(\cdot)$ is a continuous function with $\gamma(0)=0$, $\gamma^{\prime}(0)=0$, these together with $c_{n}>0$ and $\lim _{n \rightarrow \infty} c_{n}=0$ imply

$$
\lim _{n \rightarrow \infty} \frac{\left|\gamma\left(c_{n}\right)\right|}{\ln \left(1+c_{n}\right)}=0
$$

since

$$
\lim _{s \rightarrow 0^{+}} \frac{|\gamma(s)| / s}{(\ln (1+s)) / s}=0
$$

Thus

$$
\frac{\left|\gamma\left(c_{n}\right)\right|}{\ln \left(1+c_{n}\right)} 2 \pi\|h\|_{\infty}\|\phi\|_{\infty}\left\|g^{\prime}\right\|_{L^{\infty}\left(I_{0}\right)} \leq \frac{\hat{\delta}}{4}, \quad n \geq N_{1},
$$

that is,

$$
\left|\frac{J_{n}-I_{n}}{c_{n} \ln \left(1+c_{n}\right)}\right| \leq \frac{\hat{\delta}}{4}, \quad n \geq N_{1}
$$

i.e.,

$$
\frac{I_{n}}{c_{n} \ln \left(1+c_{n}\right)}-\frac{\hat{\delta}}{4} \leq \frac{J_{n}}{c_{n} \ln \left(1+c_{n}\right)} \leq \frac{I_{n}}{c_{n} \ln \left(1+c_{n}\right)}+\frac{\hat{\delta}}{4}, \quad n \geq N_{1} .
$$


From (H1), we have that there exists $N^{*}>0, \forall n \geq N^{*}$,

$$
\frac{I_{n}}{c_{n} \ln \left(1+c_{n}\right)} \geq \frac{\hat{\delta}}{2}
$$

and subsequently,

$$
\frac{J_{n}}{c_{n} \ln \left(1+c_{n}\right)} \geq \frac{I_{n}}{c_{n} \ln \left(1+c_{n}\right)}-\frac{\hat{\delta}}{4}=\frac{\hat{\delta}}{4}>0 .
$$

Thus, for $n \geq \max \left\{N_{1}, N^{*}\right\}$, we have

$$
\int_{0}^{2 \pi} h(t) g\left(c_{n} \phi(t)+\gamma\left(c_{n}\right)\right) \phi(t) d t>0
$$

However, this contradicts (2.9).

\section{Second turn of component and proof of Theorem 1.1}

In this section, we show that there is a direction turn of the bifurcation under condition (H3), and accordingly we finish the proof of Theorem 1.1.

Lemma 3.1 Let $(\mathrm{H} 1)$ and $(\mathrm{H} 2)$ hold. Assume that $\left\{\left(\lambda_{k}, u_{k}\right)\right\}$ is a sequence of positive solutions of (1.1). Assume that $\left|\lambda_{k}\right|<C_{0}$ for some constant $C_{0}>0$, and

$$
\lim _{k \rightarrow \infty}\left\|u_{k}\right\| \rightarrow \infty
$$

then

$$
\lim _{k \rightarrow \infty}\left\|u_{k}\right\|_{\infty} \rightarrow \infty
$$

Proof By Rolle's theorem, there exists $\eta \in(0,2 \pi)$ such that $u^{\prime}(\eta)=0$. Integrating the equation of $(1.1)$ over $[\eta, x]$, we have

$$
u_{k}^{\prime}(x)=\int_{\eta}^{x} u_{k}^{\prime \prime}(s)=\int_{\eta}^{x} q(s) u_{k}(s) d s-\lambda_{k} \int_{\eta}^{x} h(s) f\left(u_{k}(s)\right) d s, \quad s \in[0,2 \pi] .
$$

Recalling (1.7) for some $N>0$, we get

$$
\begin{aligned}
u_{k}^{\prime}(x) & =\int_{\eta}^{x} u_{k}^{\prime \prime}(s) \leq \int_{\eta}^{x} q(s) u_{k}(s) d s+\lambda_{k} \int_{\eta}^{x}|h(s)| f\left(u_{k}(s)\right) d s \\
& \leq\left(\int_{0}^{2 \pi} q(s) d s+C_{0} N \int_{0}^{2 \pi}|h(s)| d s\right)\left\|u_{k}\right\|_{\infty} .
\end{aligned}
$$

(3.1) implies that $\left\{\left\|u_{k}^{\prime}\right\|_{\infty}\right\}$ is bounded whenever $\left\{\left\|u_{k}\right\|_{\infty}\right\}$ is bounded.

Lemma 3.2 If $u \in D(L)$, then

$$
\left\|u^{\prime}\right\|_{\infty} \leq\left\|\left(u^{\prime \prime}\right)^{ \pm}\right\|_{L^{1}(0,2 \pi)^{\circ}} .
$$


Proof By Rolle's theorem, there exists $\eta \in(0,2 \pi)$ such that $u^{\prime}(\eta)=0$. For every $t \in[0,2 \pi]$, we have

$$
u^{\prime}(t)=\int_{\eta}^{t} u^{\prime \prime}(s) d s \leq \int_{\eta}^{t}\left(u^{\prime \prime}(s)\right)^{+} d s \leq \int_{0}^{2 \pi}\left(u^{\prime \prime}(s)\right)^{+} d s=\left\|\left(u^{\prime \prime}\right)^{+}\right\|_{L^{1}(0,2 \pi)},
$$

that is,

$$
\left\|u^{\prime}\right\|_{\infty} \leq\left\|\left(u^{\prime \prime}\right)^{+}\right\|_{L^{1}(0,2 \pi)} .
$$

On the other hand,

$$
\begin{aligned}
-u^{\prime}(t) & =\int_{\eta}^{t}-u^{\prime \prime}(s) d s \leq \int_{\eta}^{t}\left(-u^{\prime \prime}(s)\right)^{+} d s=\int_{\eta}^{t}\left(u^{\prime \prime}(s)\right)^{-} d s \leq \int_{0}^{2 \pi}\left(u^{\prime \prime}(s)\right)^{-} d s \\
& =\left\|\left(u^{\prime \prime}\right)^{-}\right\|_{L^{1}(0,2 \pi)^{\prime}}
\end{aligned}
$$

i.e.,

$$
\left\|u^{\prime}\right\|_{\infty} \leq\left\|\left(u^{\prime \prime}\right)^{-}\right\|_{L^{1}(0,2 \pi)} .
$$

Thus,

$$
\left\|u^{\prime}\right\|_{\infty} \leq\left\|\left(u^{\prime \prime}\right)^{ \pm}\right\|_{L^{1}(0,2 \pi)^{*}} .
$$

Lemma 3.3 Let $J \subseteq(0, \beta]$ be a compact interval. Assume that $(\mathrm{F} 1)$ and $(\mathrm{H} 5)$ hold. Let $(\lambda, u)$ be a positive solution of (1.1) with $\lambda \in J, u>0$. Then

$$
\frac{1}{2}\|u\|_{\infty} \leq u(t) \leq\|u\|_{\infty}, \quad t \in[0,2 \pi] .
$$

Proof We claim that if $(\lambda, u)$ is a positive solution of (1.1) with $\|u\|_{\infty}=2 s_{0}$, then

$$
\min _{t \in[0,2 \pi]} u(t) \geq s_{0} .
$$

Assume to the contrary that there exists $\left(\mu_{n}, u_{n}\right)$, a solution of problem (1.1), such that $\left\|u_{n}\right\|_{\infty}=2 s_{0}, \min _{t \in[0,2 \pi]} u_{n}(t) \rightarrow 0^{+}(n \rightarrow \infty)$.

Since $u_{n}$ is bounded in $C^{2}[0,2 \pi]$, after taking a subsequence if necessary, we have $\left\{u_{n}\right\}$ uniformly converges to a limit $\hat{u}$ with $\|\hat{u}\|_{\infty}=2 s_{0}$ and $\hat{u}\left(t_{*}\right)=0, \hat{u}^{\prime}\left(t_{*}\right)=0$ for some $t_{*} \in$ $[0,2 \pi]$. From Lemma 4.7 of [6], we know that $u(t) \equiv 0, t \in[0,2 \pi]$. This is a contradiction.

Let $\omega(u)$ be the amplitude of the solution $u$. Combining this with Lemma 3.2, we have

$$
\begin{aligned}
\omega(u) & =\int_{t_{1}}^{t_{0}} u^{\prime}(s) d s \leq 2 \pi\left\|u^{\prime}\right\|_{\infty} \leq 2 \pi\left\|\left(u^{\prime \prime}\right)^{+}\right\|_{L^{1}(0,2 \pi)}=2 \pi \int_{0}^{2 \pi}\left(u^{\prime \prime}\right)^{+} d s \\
& =2 \pi \int_{0}^{2 \pi}\left(-u^{\prime \prime}\right)^{-} d s .
\end{aligned}
$$

Here, $u\left(t_{0}\right)=\|u\|_{\infty}, u\left(t_{1}\right)=\min _{t \in[0,2 \pi]} u(t)$. 
By simple computation, we get

$$
\begin{aligned}
\omega(u) & \leq 2 \pi \int_{0}^{2 \pi}\left(-u^{\prime \prime}\right)^{-} d s \\
& =2 \pi\left\|(\lambda h(t) f(u)-q(t) u)^{-}\right\|_{L^{1}(0,2 \pi)} \\
& \leq 2 \pi\left\|\lambda h^{-}(t) f(u)\right\|_{L^{1}(0,2 \pi)}+2 \pi\|q(t) u\|_{L^{1}(0,2 \pi)} .
\end{aligned}
$$

Since for any function $a(x), b(x)$, we have

$$
[a(x)+b(x)]^{-} \leq a^{-}(x)+b^{-}(x) .
$$

Combining this with (H5), we have

$$
\omega(u) \leq s_{0}
$$

Thus

$$
\min _{t \in[0,2 \pi]} u(t) \geq s_{0}=\frac{1}{2}\|u\|_{\infty} .
$$

Therefore, the claim is proved.

Lemma 3.4 Assume that (F1) and (H4) hold. Let $u$ be a positive solution of (1.1) with $\|u\|_{\infty}=u\left(t_{0}\right)$, then for every $\lambda>\beta$, we have

$$
\frac{1}{2}\|u\|_{\infty} \leq u(t) \leq\|u\|_{\infty}, \quad t \in\left[\frac{t_{0}+x_{2 i_{0}-1}}{2}, \frac{t_{0}+x_{2 i_{0}}}{2}\right]
$$

and $t_{0} \in\left(x_{2 i_{0}-1}, x_{2 i_{0}}\right)$ for some $i_{0} \in\{1,2, \ldots, n\}$.

Proof Since $-u^{\prime \prime}(t)=\left(\lambda h(t) \frac{f(u)}{u}-q(t)\right) u$, condition $(\mathrm{H} 4)$ implies that

$$
-u^{\prime \prime}(t) \geq \beta h^{+}(t) \inf \left\{\frac{f(u)}{u}: u \in\left(0,2 s_{0}\right]\right\} u(t)-q(t) u(t) \geq 0, \quad t \in \bigcup_{i=1}^{n}\left(x_{2 i-1}, x_{2 i}\right)
$$

Note that condition (F1) implies that $u^{\prime}(t)$ is decreasing on $\bigcup_{i=1}^{n}\left(x_{2 i-1}, x_{2 i}\right)$. Moreover, if $t \in[0,2 \pi] \backslash \bigcup_{i=1}^{n}\left[x_{2 i-1}, x_{2 i}\right],(\mathrm{F} 1)$ implies that $u^{\prime}(t)$ is increasing on $[0,2 \pi] \backslash \bigcup_{i=1}^{n}\left[x_{2 i-1}, x_{2 i}\right]$. Thus $u$ is convex on $[0,2 \pi] \backslash \bigcup_{i=1}^{n}\left[x_{2 i-1}, x_{2 i}\right]$ and concave on $\bigcup_{i=1}^{n}\left(x_{2 i-1}, x_{2 i}\right)$. Therefore, $t_{0}$ must be in $\left(x_{2 i_{0}-1}, x_{2 i_{0}}\right)$ for some $i_{0} \in\{1,2, \ldots, n\}$.

In fact, assume on the contrary that $t_{0} \in[0,2 \pi] \backslash \bigcup_{i=1}^{n}\left[x_{2 i-1}, x_{2 i}\right]$, then it follows from

$$
-u^{\prime \prime}\left(t_{0}\right)+q\left(t_{0}\right) u\left(t_{0}\right)=\lambda h\left(t_{0}\right) f\left(u\left(t_{0}\right)\right) .
$$

Since $f\left(u\left(t_{0}\right)\right)>0, h\left(t_{0}\right)<0$, and $\lambda>0$, we have that the right-hand side is negative. However, $q\left(t_{0}\right) u\left(t_{0}\right) \geq 0$ and $u^{\prime \prime}\left(t_{0}\right) \leq 0$ imply that the left-hand side is nonnegative. This is a contradiction. Therefore,

$$
t_{0} \in\left(x_{2 i_{0}-1}, x_{2 i_{0}}\right) .
$$


By simple computation, we get

$$
u(t) \geq \frac{\|u\|_{\infty}}{t_{0}-x_{2 i_{0}-1}}\left(t-x_{2 i_{0}-1}\right), \quad t \in\left[x_{2 i_{0}-1}, t_{0}\right]
$$

Note that $\frac{t-x_{2 i_{0}-1}}{t_{0}-x_{2 i_{0}-1}} \geq \frac{1}{2}$ is equivalent to $t \geq \frac{t_{0}+x_{2 i_{0}-1}}{2}$. Similarly,

$$
u(t) \geq \frac{\|u\|_{\infty}}{x_{2 i_{0}}-t_{0}}\left(x_{2 i_{0}}-t\right), \quad t \in\left[t_{0}, x_{2 i_{0}}\right]
$$

and $\frac{x_{2 i_{0}}-t}{x_{2 i_{0}}-t_{0}} \geq \frac{1}{2}$ is equivalent to $t \leq \frac{t_{0}+x_{2 i_{0}}}{2}$. Therefore, we have

$$
\frac{1}{2}\|u\|_{\infty} \leq u(t) \leq\|u\|_{\infty}, \quad t \in\left[\frac{t_{0}+x_{2 i_{0}-1}}{2}, \frac{t_{0}+x_{2 i_{0}}}{2}\right]
$$

Lemma 3.5 Assume that (F1) and (H4) hold. Let $u$ be a positive solution of (1.1) with $\|u\|_{\infty}=2 s_{0}$. Then $\lambda<\frac{\lambda_{0}^{+}}{f_{0}}$.

Proof Let $u$ be a positive solution of (1.1) with $\|u\|_{\infty}=2 s_{0}$. By Lemmas 3.3, 3.4,

$$
s_{0} \leq u(t) \leq 2 s_{0}, \quad t \in I
$$

where

$$
I=\left[\frac{t_{0}+x_{2 i_{0}-1}}{2}, \frac{t_{0}+x_{2 i_{0}}}{2}\right]
$$

Now we assume $\lambda \geq \frac{\lambda_{0}^{+}}{f_{0}}$. Then, for $t \in I$, by (H3), we get

$$
\lambda h(t) \frac{f(u(t))}{u(t)}-q(t) \geq \frac{\lambda_{0}^{+}}{f_{0}} h_{0} \frac{f_{0}}{\lambda_{0}^{+} h_{0}}\left[\left(\frac{2 \pi}{l}\right)^{2}+\hat{q}\right]-\hat{q}=\left(\frac{2 \pi}{l}\right)^{2} \geq\left(\frac{2 \pi}{x_{2 i_{0}}-x_{2 i_{0}-1}}\right)^{2} .
$$

Set

$$
v(t):=\sin \left(\frac{2 \pi}{x_{2 i_{0}}-x_{2 i_{0}-1}}\left(t-\frac{t_{0}+x_{2 i_{0}-1}}{2}\right)\right),
$$

it is known that $v\left(\frac{t_{0}+x_{2 i_{0}-1}}{2}\right)=v\left(\frac{t_{0}+x_{2 i_{0}}}{2}\right)=0$.

Then $v$ is a solution of

$$
\left\{\begin{array}{l}
v^{\prime \prime}(t)+\left(\frac{2 \pi}{x_{2 i_{0}}-x_{2 i_{0}-1}}\right)^{2} v(t)=0, \quad t \in I \\
v\left(\frac{t_{0}+x_{2 i_{0}-1}}{2}\right)=v\left(\frac{t_{0}+x_{2 i}}{2}\right)=0
\end{array}\right.
$$

Obviously $v(t)>0$ on $\left(\frac{t_{0}+x_{2 i_{0}-1}}{2}, \frac{t_{0}+x_{2 i_{0}}}{2}\right)$.

We note that $u$ is a solution of

$$
u^{\prime \prime}(t)+\left(\lambda h(t) \frac{f(u(t))}{u(t)}-q(t)\right) u(t)=0
$$


on $I$. The Sturm comparison theorem [16] implies that $u$ has at least one zero on $I$. This contradicts the fact that $u(t)>0$ on $I$.

Using an argument similar to proving [6, Theorem 5.2] with obvious changes, we have the following lemma.

Lemma 3.6 If $f_{0} \in(0,+\infty)$ and $f_{\infty}=0$, then $\mathcal{C}^{+}$joins $\left(\frac{\lambda_{0}^{+}}{f_{0}}, 0\right)$ to $(+\infty,+\infty), \mathcal{C}^{-}$joins $\left(\frac{\lambda_{0}^{-}}{f_{0}}, 0\right)$ to $(-\infty,+\infty)$.

Proof of Theorem 1.1 From Lemma 2.1, there exists an unbounded connected component $\mathcal{C}^{v}$ in the positive solutions set of (1.1). Moreover, $\mathcal{C}^{v} \subset\left(\left(\mathbb{R}^{+} \times \operatorname{int} P\right) \cup\left\{\frac{\lambda_{0}^{v}}{f_{0}}, 0\right\}\right)$, where $v \in$ $\{+,-\}$.

By Lemma 3.1, it follows that there exists $\left(\lambda_{0}, u_{0}\right) \in \mathcal{C}^{+}$such that $\left\|u_{0}\right\|_{\infty}=2 s_{0}$ and Lemma 3.5 implies that $\lambda_{0}<\frac{\lambda_{0}^{+}}{f_{0}}$. By Lemmas $2.4,3.5, \mathcal{C}^{+}$passes through some points $\left(\frac{\lambda_{0}^{+}}{f_{0}}, v_{1}\right)$ and $\left(\frac{\lambda_{0}^{+}}{f_{0}}, v_{2}\right)$ with $\left\|v_{1}\right\|_{\infty}<2 s_{0}<\left\|v_{2}\right\|_{\infty}$, and there exist $\underline{\lambda}$ and $\bar{\lambda}$ which satisfy $0<\underline{\lambda}<\frac{\lambda_{0}^{+}}{f_{0}}<\bar{\lambda}$ and both (i) and (ii):

(i) If $\lambda \in\left(\frac{\lambda_{0}^{+}}{f_{0}}, \bar{\lambda}\right]$, then there exist $u$ and $v$ such that $(\lambda, u),(\lambda, v) \in \mathcal{C}^{+}$and $\|u\|_{\infty}<\|v\|_{\infty}<2 s_{0}$

(ii) If $\lambda \in\left(\underline{\lambda}, \frac{\lambda_{0}^{+}}{f_{0}}\right]$, then there exist $u$ and $v$ such that $(\lambda, u),(\lambda, v) \in \mathcal{C}^{+}$and $\|u\|_{\infty}<2 s_{0}<\|v\|_{\infty}$.

Define $\lambda^{*}=\sup \{\bar{\lambda}: \bar{\lambda}$ satisfies (i) $\}$ and $\lambda_{*}=\inf \{\underline{\lambda}: \underline{\lambda}$ satisfies (ii) $\}$. Then (1.1) has a positive solution $u_{\lambda_{*}}$ at $\lambda=\lambda_{*}$ and $u_{\lambda^{*}}$ at $\lambda=\lambda^{*}$, respectively.

Clearly, $\mathcal{C}^{+}$turns to the left at $\left(\lambda^{*},\left\|u_{\lambda^{*}}\right\|_{\infty}\right)$ and to the right at $\left(\lambda_{*},\left\|u_{\lambda_{*}}\right\|_{\infty}\right)$, finally to the right near $\lambda=+\infty$. Furthermore, by Lemma 3.6, it follows that, for each $\lambda \in\left(-\infty, \frac{\lambda_{0}^{-}}{f_{0}}\right)$, problem (1.1) has a positive solution. This completes the proof of Theorem 1.1.

Now we strengthen the assumptions on $f$ and $h$ as follows:

(H6) $f \in C^{2}([0, \infty),[0, \infty))$ with $f(0)=0$ and $f(s)>0$ for $s>0$;

(H7) $f^{\prime \prime}(s)<0$ for $s \in\left[0, s_{1}\right)$;

(H8) $h \in C([0,2 \pi],[0,+\infty))$ and $h \not \equiv 0$.

By Theorem 1.1, we can easily show the following corollary.

Corollary 3.1 Assume that (H2), (H3), (H6)-(H8) hold. Then there exist $\lambda_{*} \in\left(0, \frac{\lambda_{0}^{+}}{f_{0}}\right)$ and $\lambda^{*}>\frac{\lambda_{0}^{+}}{f_{0}}$ such that

(i) (1.1) has at least one positive solution if $\lambda=\lambda_{*}$;

(ii) (1.1) has at least two positive solutions if $\lambda_{*}<\lambda \leq \frac{\lambda_{0}^{+}}{f_{0}}$;

(iii) (1.1) has at least three positive solutions if $\frac{\lambda_{0}^{+}}{f_{0}}<\lambda<\lambda^{*}$;

(iv) (1.1) has at least two positive solutions if $\lambda=\lambda^{*}$;

(v) (1.1) has at least one positive solution if $\lambda>\lambda^{*}$;

(vi) (1.1) has at least one positive solution if $\lambda<\frac{\lambda_{0}^{-}}{f_{0}}$.

Remark 3.1 Let us consider the nonlinear problem

$$
\left\{\begin{array}{l}
-u^{\prime \prime}(t)+q_{\sigma}(t) u(t)=\lambda h_{\sigma}(t) f(u(t)), \quad t \in(0,1) \\
u(0)=u(1), \quad u^{\prime}(0)=u^{\prime}(1)
\end{array}\right.
$$


where $\sigma \in\left(0, \frac{1}{2}\right)$ is a parameter,

$$
\begin{aligned}
& h_{\sigma}(t)=1-t-\sigma, \quad t \in[0,1], \\
& q_{\sigma}(t)=\frac{1}{10} \begin{cases}1-t-\sigma, & t \in[0,1-\sigma], \\
t-1+\sigma, & t \in(1-\sigma, 1],\end{cases} \\
& f(s)= \begin{cases}s-s \ln (1+s), & s \in[0,1), \\
\kappa s-\kappa+1-\ln 2, & s \in[1,4), \\
\sqrt{s}+3 \kappa-1-\ln 2, & s \in[4, \infty) .\end{cases}
\end{aligned}
$$

Here $\kappa=\frac{80 \pi^{2}}{(1-\sigma)^{2}}+2$.

Let $\lambda_{0}^{+}(\sigma)$ be the first positive eigenvalue corresponding to the linear problem

$$
\left\{\begin{array}{l}
-u^{\prime \prime}(t)+q_{\sigma} u(t)=\lambda h_{\sigma}(t) u(t), \quad t \in(0,1) \\
u(0)=u(1), \quad u^{\prime}(0)=u^{\prime}(1)
\end{array}\right.
$$

Let $\phi$ be the positive eigenfunction corresponding to $\lambda_{0}^{+}(\sigma)$. Next, we will estimate the range of the value of $\lambda_{0}^{+}(\sigma)$. Recalling

$$
\lambda_{0}^{+}(\sigma)=\inf \left\{\frac{\int_{0}^{1}\left(\left(u^{\prime}\right)^{2}+q_{\sigma} u^{2}\right) d t}{\int_{0}^{1} h_{\sigma} u^{2} d t} \mid u(0)=u(1), u^{\prime}(0)=u^{\prime}(1)\right\} .
$$

Since

$$
\frac{\int_{0}^{1}\left(\left(u^{\prime}\right)^{2}+q_{\sigma} u^{2}\right) d t}{\int_{0}^{1} h_{\sigma} u^{2} d t} \geq \frac{\int_{0}^{1} q_{\sigma} u^{2} d t}{\int_{0}^{1} h_{\sigma} u^{2} d t} \geq \frac{\int_{0}^{1-\sigma} q_{\sigma} u^{2} d t}{\int_{0}^{1-\sigma} h_{\sigma} u^{2} d t}=\frac{1}{10},
$$

that is,

$$
\lambda_{0}^{+}(\sigma) \geq \frac{1}{10} .
$$

We will check that all of the conditions in Theorem 1.1 are fulfilled. In fact,

$$
f_{0}=1 ; \quad g(s)=s \ln (1+s) \quad \text { for } s \in[0,1],
$$

and

$$
\lim _{c \rightarrow 0^{+}} \int_{0}^{1} h_{\sigma}(s) \frac{g(c \phi(s))}{c \ln (1+c)} \phi(s) d s=\int_{0}^{1} h(s) \phi^{3}(s) d s=\Delta>0
$$

(see Lemma 2.3).

Let $\|u\|_{\infty}=4$, then the function $f$ satisfies (H3) with $s_{0}=2, l=1-\sigma, \hat{q_{\sigma}}=\frac{1}{10}(1-\sigma)$, $h_{\sigma 0}=\frac{1-\sigma}{2}$, such that

$$
\min _{s \in[2,4]} \frac{f(s)}{s} \geq \frac{80 \pi^{2}}{(1-\sigma)^{2}}+2 .
$$

Furthermore, the function $f$ satisfies (H1) and (H2). 
Moreover,

$$
\begin{aligned}
& \inf \left\{\frac{f(s)}{s}: s \in(0,4]\right\}=1-\ln 2, \quad \max \{f(s): s \in[0,4]\}=3 \kappa+1-\ln 2, \\
& h_{\sigma}^{+}(t)= \begin{cases}1-t-\sigma, & t \in[0,1-\sigma], \\
0, & t \in(1-\sigma, 1],\end{cases} \\
& h_{\sigma}^{-}(t)= \begin{cases}0, & t \in[0,1-\sigma], \\
t-1+\sigma, & t \in(1-\sigma, 1] .\end{cases}
\end{aligned}
$$

Note that $\left\|h_{\sigma}^{-}\right\|_{L^{1}(0,1)}=\frac{1}{2} \sigma^{2},\left\|q_{\sigma}\right\|_{\infty}=\frac{1-\sigma}{10}$. Obviously, there exists $\beta=4$ such that (H4) and (H5) are satisfied with $\sigma \leq \sigma_{0}$. Here, $\sigma_{0}$ is the unique positive solution of the equation

$$
2 \sigma^{2}(3 \kappa+1-\ln 2)+\frac{2(1-\sigma)}{5}=2 .
$$

Since, $\chi(\sigma):=2 \sigma^{2}(3 \kappa+1-\ln 2)+\frac{2(1-\sigma)}{5}-2$ is strictly increasing for $\sigma \in[0,1)$ with $\chi(0)=$ $-\frac{8}{5}$.

By using maple 17, we may get

$$
\sigma_{0} \approx 0.018
$$

Therefore, the conclusions of Theorem 1.1 are valid if $\sigma \leq \sigma_{0}$.

Remark 3.2 It is worth remarking that $g(s)=s \ln (1+s)$ for $s \in[0,1]$ has a big difference with the function $g(s) \approx f_{1} s^{1+\alpha}$ for some $\alpha>1$ (see [14, (F1)]) since

$$
\lim _{s \rightarrow 0^{+}} \frac{s \ln (1+s)}{s^{1+\alpha}}=\infty
$$

\section{Acknowledgements}

The authors express their sincere gratitude to the editors and anonymous referee for the careful reading of the original manuscript and useful comments that helped to improve the presentation of the results and accentuate important details.

\section{Funding}

This work was supported by the National Natural Science Foundation of China (No. 11671322).

\section{Abbreviations}

Not applicable.

\section{Availability of data and materials}

Data sharing not applicable to this article as no datasets were generated.

\section{Competing interests}

The authors declare that they have no competing interests.

Authors' contributions

RM completed the main study, carried out the results of this article, and drafted the paper. ZH and MX checked the proofs and verified the calculation. Both authors read and approved the final manuscript.

\section{Publisher's Note}

Springer Nature remains neutral with regard to jurisdictional claims in published maps and institutional affiliations. 


\section{References}

1. Graef, J.R., Kong, L., Wang, H.: Existence, multiplicity, and dependence on a parameter for a periodic boundary value problem. J. Differ. Equ. 245, 1185-1197 (2008)

2. Hao, X., Liu, L., Wu, Y.: Existence and multiplicity results for nonlinear periodic boundary value problems. Nonlinear Anal. 72, 3635-3642 (2010)

3. Ma, R., Xu, J., Han, X.: Global bifurcation of positive solutions of a second-order periodic boundary value problem with indefinite weight. Nonlinear Anal. 74, 3379-3385 (2011)

4. Boscaggin, A., Zanolin, F.: Pairs of positive periodic solutions of second order nonlinear equations with indefinite weight. J. Differ. Equ. 252, 2900-2921 (2012)

5. Hakl, R., Zamora, M.: Periodic solutions to second-order indefinite singular equations. J. Differ. Equ. 263, 451-469 (2017)

6. Dai, G., Ma, R., Wang, H.: Eigenvalues, bifurcation and one-sign solutions for the periodic $p$-Laplacian. Commun. Pure Appl. Anal. 12, 2839-2872 (2013)

7. Constantin, A.: A general-weighted Sturm-Liouville problem. Ann. Sci. Éc. Norm. Supér. (4) 24, 767-782 (1997)

8. Chu, J., Torres, P.J., Wang, F.: Twist periodic solutions for differential equations with a combined attractive-repulsive singularity. J. Math. Anal. Appl. 437, 1070-1083 (2016)

9. Lomtatidze, A., Šremr, J.: On periodic solutions to second-order Duffing type equations. Nonlinear Anal., Real World Appl. 40, 215-242 (2018)

10. Atici, F.M., Guseinov, G.Sh.: On the existence of positive solutions for nonlinear differential equations with periodic boundary conditions. J. Comput. Appl. Math. 132, 341-356 (2001)

11. Ma, R., Gao, C., Chen, R.: Existence of positive solutions of nonlinear second-order periodic boundary value problems. Bound. Value Probl. (2010). https://doi.org/10.1155/2010/626054

12. $\mathrm{Xu}, \mathrm{J}$., Ma, R.: Bifurcation from interval and positive solutions for second order periodic boundary value problems. Appl. Math. Comput. 216, 2463-2471 (2010)

13. Ma, R., Xu, J., Han, X.: Global structure of positive solutions for superlinear second-order periodic boundary value problems. Appl. Math. Comput. 218, 5982-5988 (2012)

14. Sim, I., Tanaka, S.: Three positive solutions for one-dimensional $p$-Laplacian problem with sign-changing weight. Appl. Math. Lett. 49, 42-50 (2015)

15. Crandall, M.G., Rabinowitz, P.H.: Bifurcation from simple eigenvalues. J. Funct. Anal. 8, 321-340 (1971)

16. Li, H., Yeh, C.: Sturmian comparison theorem for half-linear second-order differential equations. Proc. R. Soc. Edinb. A $125,1193-1204(1995)$

\section{Submit your manuscript to a SpringerOpen ${ }^{\circ}$ journal and benefit from:}

- Convenient online submission

- Rigorous peer review

- Open access: articles freely available online

- High visibility within the field

- Retaining the copyright to your article

Submit your next manuscript at $>$ springeropen.com 